\title{
THE IMPACT OF MOVING THROUGH THE BUILT ENVIRONMENT ON EMOTIONAL AND NEUROPHYSIOLOGICAL STATE
}

\author{
A Systematic Literature Review
}

\author{
MITRA HOMOLJA ${ }^{1}$, SAYYED AMIR HOSSAIN MAGHOOL ${ }^{2}$ and \\ MARC AUREL SCHNABEL ${ }^{3}$ \\ $1,2,3$ Victoria University of Wellington \\ ${ }^{1}$ Mitra.Homolja@gmail.com ${ }^{2,3}$ \{SayyedAmirHossain.Maghool| \\ MarcAurel.Schnabel\}@vuw.ac.nz
}

\begin{abstract}
Despite theoretical evidence about the capabilities of visual properties of space for manipulating inhabitants' emotions, a gap in knowledge exists for empirical studies in controlled environments. Interdisciplinary studies at the intersection of architecture, psychology, and neuroscience can provide robust guidelines and criteria for designers to engineer emotions. Due to the novelty of the field, the theoretical framework for such studies is not well established. Consequently, this paper presents a systematic literature review to find and synthesize recent relevant studies at this intersection. Based on these findings, we will investigate the impact of other visuo-spatial stimuli on emotions in a rigorous way. According to the theories of emotions, manipulation of emotions is linked to oscillations in physiological responses caused by exposure to sensory stimuli. Moreover, there is a consensus that human perception is action-oriented. Therefore, our review focuses on studies that employ biosensors as subjects move in physical or virtual environments.
\end{abstract}

Keywords. Neuroarchitecture; Brain Body Sensors; Virtual Reality; Physiological Response; Emotional Response.

\section{Introduction}

The qualities of spaces in the built environment impact profoundly on how we exist within them. Our perception of architecture and the built environment affects our physiological and emotional experiences of our immediate surroundings. There is a consensus that sensory stimuli in the built environment impact our feelings and emotions; however, the interrelation between architecture and the emotions it evokes is not yet fully understood. A gap exists in the knowledge at the intersection of architecture, psychology, and neuroscience. The intersection's relevance is gaining traction, but the conclusions on the matter are not straightforward, nor imminent. Moreover, there is an inherent need for architects to understand how their buildings impact the emotions of those who occupy them; for instance,

RE: Anthropocene, Proceedings of the $25^{\text {th }}$ International Conference of the Association for Computer-Aided Architectural Design Research in Asia (CAADRIA) 2020, Volume 1, 641-650. (C) 2020 and published by the Association for Computer-Aided Architectural Design Research in Asia (CAADRIA), Hong Kong. 
engineering people's emotional states when designing a memorial or a sacred building (Eberhard 2008). As Kahn states, "one may say that architecture is the thoughtful making of spaces. It is not the filling of areas proscribed by a client. It is the creating of spaces that evoke a feeling of appropriate use" (Kahn $\&$ Brillembourg 1992).

This paper is a part of a research project that aims to study the impact of variation in visuo-spatial variables on humans while they walk through built environments. We will utilize technologies, namely virtual reality and human body-brain sensors, to rigorously study the impact of the variation on subjective and objective emotional data gathered from human subjects. Our paper consists of a systematic literature review, where we identify existing research in the field of neuroarchitecture and environmental psychology. We intend to discuss contemporary empirical literature with the aim of presenting a compelling argument in favor of the need for further research in this field.

In order to understand the effects of spatial properties on human emotion, we have selected empirical studies that use subjective and objective measurements. Additionally, we identified studies that consider the importance of movement in the perception and experience of architectural space. We synthesise current literature to elucidate gaps in the existing knowledge and to establish our trajectory for research in this field.

\section{Background}

Many researchers claim that we are at the beginning of a revolution in cognitive research and neuroscience; they suggest it is comparable to the Galilean revolution in physics (Robinson 2015). A growing body of research explores the impacts neuroscience can have on the field of architecture to offer designers novel possibilities for architecture with intent to impact people's short-term and long-term emotional states. A branch of science called neuroarchitecture is emerging from these studies (Banaei et al. 2017), where modern methodologies are employed from several disciplines into research in the architecture field.

Numerous studies have noted that emphatically measuring emotion is difficult; quantifying a very subjective emotive experience is an inherently complex task and subjective methodologies alone are argued to have limited effectiveness. Eberhard (2009) suggests that we can use metaphors to convey a part of emotions being experienced by our consciousness, but they are not the real experience of places. It emphasizes the importance of understanding subconscious neurophysiological reactions of experienced emotions. Neuroscience and psychology have developed a body of research which attempts to address the nature of primal responses to our surroundings; the James-Lange theory (James 1884), Cannon-Bard theory (Cannon 1927), Schachter-Singer theory (Schachter \& Singer 1962), Facial Feedback theory (Lanzetta et al. 1976), all emphasize that external stimuli that trigger emotional responses are closely related to autonomous physiological responses.

Emotion is reflected in all modes of human communication such as word choice, tone of voice, facial expression, gestural behaviour, posture, skin 


\section{THE IMPACT OF MOVING THROUGH THE BUILT ENVIRONMENT ON EMOTIONAL AND NEUROPHYSIOLOGICAL STATE}

temperature and clamminess, respiration, muscle tension, and more (Picard et al. 2001). Picard et al. (2001) elaborate on how emotional recognition systems are most likely to be accurate when they combine "multiple kinds of signals from the user with information about the user's context, situation, goals, and preferences." They suggest "a combination of low-level pattern recognition, high-level reasoning, and natural language processing is likely to provide the best emotion inference" (Picard et al. 2001 p.1176). Consequently, we sought out studies that use any biosensors to measure biodata from subjects objectively.

Many have discussed the importance of the sense of movement in the architecture of historical buildings for evoking senses, such as a sense of awe. Barrie (1996) suggests that surface and textures, scale and distance of entrances, the shifting view as we move along the route, inclines and steps, our peripheral field of vision, and our empathy with the environment (reflected through decorations) are visuo-spatial factors that impact our emotions, and are the essential components of the experience of architecture.

Bruno discusses the interconnection between "sight" and "site" and "motion" and "emotion" while describing emotions in architecture and other arts (Bruno 2007). This means that studies concerned about emotion and architecture should approach architecture as an experience with a "duration" and a "path of movement". It stems from the notion that perception is action-oriented (Gibson 1966). By eliminating movement, studies may be limiting a real exploration of primal physiological responses (Schreuder et al. 2016). We elucidate a research trajectory which can build on existing knowledge in the field with the intention of addressing how movement in a controlled virtual test environment impacts on the physiological responses of participants.

\section{Method}

\subsection{SEARCH PROCEDURE}

A systematic literature review was conducted in order to identify studies relevant to the search objectives and to identify the scope for future research. Due to the recently emerging research in regards to the built environment's impact on neurophysiological responses, the literature review's search terms, exclusion criteria and inclusion criteria required an iterative systematic approach to refine search terms and identify relevant material. There tends to be a discrepancy between recording objective measurements of physiological responses and correlating it as conclusive data of states of "emotion". Due to the limited amount of empirical research in the field, studies which used combined methods that recorded both subjective and objective human responses were considered for examination.

The following inclusion criteria were implemented to ensure the parameters of the literature search were relevant and within the scope of the review. Studies must include the use of objective and subjective methods of recording human physiological responses and experiences. Studies which reported empirical data were included, and studies which were purely theoretical were excluded after screening. The search was limited to investigations of physiological variables 
related to the "built environment," thus excluding social, cultural and behavioural factors and "clinical" studies. Studies should include some form of the virtual environment (VE) such as VR, CAVE, AR or XR as the controlled study environment, but the scope is open to physical spaces or other multisensory media (pictures, films, etc.). Studies should use monitoring equipment such as Electroencephalogram (EEG), Electrocardiogram (ECG), Functional Magnetic Resonance Imaging (fMRI), Galvanic Skin Response (GSR), or other for objective measurements. Studies should include questionnaires, surveys, Self-Assessment Manikins (SAM), Likert scales, verbal responses or other as a form of subjective self-evaluative response. Studies must have participants which are "human" and must exclude "animal" participants. Studies must exclude participants who are considered to be "children" or "elderly". Due to the novelty of the field, in conjunction with technology's rapid refinement, the search was limited to studies published between January 1st 2010 and January 1st 2020. Studies were limited to publications which were peer-reviewed. Studies were limited to publications in English.

\subsection{ELECTRONIC SEARCH STRATEGY}

Two electronic databases (ProQuest and Scopus) were identified as dependable directories for empirical studies as both provide advanced search option with the ability to refine inclusion and exclusion criteria. We used the advanced search option available in the electronic databases and implemented the eligibility criteria as was formerly established. Searches were conducted with an established set of keywords and refined through iterations. We screened results based on their titles and abstracts until the results which appeared were no longer relevant to the review. Citations were reviewed of articles which were deemed eligible in order to ensure other relevant referenced studies were identified.

\subsection{SEARCH TERMS RATIONALE}

A similar strategy was implemented for both ProQuest and Scopus and was adjusted to their individual syntax configurations and search restrictions. We identified three relevant groups of search terms: (1) keywords related to the experiments, stimuli, and design variables that are useful for finding studies related to architecture and the built environment; (2) keywords related to "methodologies" for studying human emotional and neurophysiological responses to those stimuli; and (3) keywords related to scientific terms for humans' responses to external stimuli and the different ways that humans reveal their conscious and unconscious processes. Within each group, we used the "OR" function to expand the search result. Between the groups, we used the "AND" function to limit the number of search results, ensuring that each search result would have at least one keyword from each group of keywords. We searched for the most used keywords in the first search attempt and used them to refine the three groups of keywords. We reviewed those keywords, and the ones that were completely out of the scope of our research were selected. Those selected keywords constructed the fourth (4) keyword group consisting of terms which are to be excluded. It ensured the final results shows only the studies that include at least one keyword from each of the 
first three groups and exclude keyword from the fourth group. Resulting in that many irrelevant results were removed from the search. A number of studies were identified via a manual search by examining references of existing studies, in order to mitigate electronic database bias and limitations. We used Google Scholar, and Researchgate websites to manually review the reference list of the selected papers. We explored the articles that have cited those selected papers using tools available on those two websites. It ensured relevant material which was not identified by electronic databases could be included.

\subsection{MITIGATION OF BIAS}

The literature review involves two people refining the method and the reviewing literature to mitigate individual bias. The method was further refined with the aid of two other researchers. Additionally, the search term effectiveness was counselled and filtered with the librarians in the Victoria University of Wellington Faculty of Architecture and Design.

\section{Results}

\subsection{IDENTIFICATION OF RELEVANT STUDIES}

Initially, 3208 results (2956 from ProQuest; 252 from Scopus) were identified based on the established search terms. After a number of iterations, the keyword group (4) consisting of terms which are to be excluded was applied to the databases, resulting in 660 results (545 from ProQuest; 115 from Scopus). After the screening of titles and abstracts and the addition of manually searched studies, a final 24 studies were identified and agreed upon by the authors. These studies underwent a full-text screening, thereby categorizing the main variables in each study in order to identify similar and juxtaposing trends.

Table 1. Details of selected studies which implement "natural walking" in controlled physical

\begin{tabular}{|c|c|c|c|c|c|c|c|}
\hline AUTHOR & YEAR & $\begin{array}{l}\text { TEST } \\
\text { SETTING }\end{array}$ & $\begin{array}{l}\text { OBJECTIVE } \\
\text { MEASUREMENT } \\
\text { TOOLS }\end{array}$ & $\begin{array}{l}\text { SUBJECTIVE } \\
\text { MEASUREMENT }\end{array}$ & $\begin{array}{l}\text { DATA } \\
\text { ANALYSIS } \\
\text { TECHNIQUE }\end{array}$ & $\begin{array}{l}\text { MOBILE } \\
\text { OR NON- } \\
\text { MOBILE } \\
\text { TESTS }\end{array}$ & $\begin{array}{l}\text { INDEPENDENT } \\
\text { VARIABLES }\end{array}$ \\
\hline $\begin{array}{l}\text { BANAEI ET } \\
\text { AL. }\end{array}$ & 2017 & $\begin{array}{l}\text { Virtual } \\
\text { Reality } \\
\text { using } \\
\text { HMD }\end{array}$ & EEG 128-channel & SAM & ANOVA & Mobile & $\begin{array}{l}\text { Various } \\
\text { architectural } \\
\text { styles }\end{array}$ \\
\hline $\begin{array}{l}\text { CHEN ET } \\
\text { AL. }\end{array}$ & 2018 & $\begin{array}{l}\text { Physical } \\
\text { Urban } \\
\text { Space }\end{array}$ & $\begin{array}{l}\text { EEG / ECG / EMG / } \\
\text { GSR }\end{array}$ & $\begin{array}{l}\text { Interview and self- } \\
\text { report of } \\
\text { physiological state }\end{array}$ & Qualitative & Mobile & $\begin{array}{l}\text { Properties of the } \\
\text { surrounding } \\
\text { environment }\end{array}$ \\
\hline $\begin{array}{l}\text { DJEBBARA } \\
\text { ET AL. }\end{array}$ & 2019 & $\begin{array}{l}\text { Virtual } \\
\text { Reality } \\
\text { using } \\
\text { HMD }\end{array}$ & EEG 64-channel & SAM & ANOVA & Mobile & $\begin{array}{l}\text { Size of entrance } \\
\text { between two } \\
\text { rooms }\end{array}$ \\
\hline $\begin{array}{l}\text { OJHA ET } \\
\text { AL. }\end{array}$ & 2019 & $\begin{array}{l}\text { Physical } \\
\text { Urban } \\
\text { Space }\end{array}$ & EEG / GSR & N/A & $\begin{array}{l}\text { REP-Tree } \\
\text { decision tree }\end{array}$ & Mobile & $\begin{array}{l}\text { "Normal" VS } \\
\text { "Aroused" } \\
\text { physiological } \\
\text { conditions }\end{array}$ \\
\hline YIN ET AL. & 2019 & $\begin{array}{l}\text { Virtual } \\
\text { Reality } \\
\text { using } \\
\text { HMD }\end{array}$ & $\begin{array}{l}\text { ECG / GSR / Eye } \\
\text { Tracker / Pulse } \\
\text { rate / Blood } \\
\text { Pressure }\end{array}$ & Questionnaires & ANOVA & Mobile & $\begin{array}{l}\text { Open space VS } \\
\text { enclosed space }\end{array}$ \\
\hline $\begin{array}{l}\text { HORVAT } \\
\text { ET AL. }\end{array}$ & 2019 & $\begin{array}{l}\text { Virtual } \\
\text { Reality } \\
\text { using } \\
\text { HMD }\end{array}$ & EEG 14-channel & N/A & $\begin{array}{l}\text { Pearson's } \\
\text { Correlation } \\
\text { Coefficient }\end{array}$ & Mobile & $\begin{array}{l}\text { Virtual } \\
\text { environments with } \\
\text { fearful images VS } \\
\text { happy images }\end{array}$ \\
\hline
\end{tabular}




\subsection{IDENTIFICATION OF STUDIES USING MOBILE NAVIGATION}

Our particular interest lies in how moving through a space impacts on human physiological responses and, in turn, their perception of space. From our full-text screening, a number of studies enabled participants to be mobile in the test scenario. The factor leads to the removal of 18 papers, as only six studies tested participants navigating physical or virtual environments by natural walking (Banaei et al. 2017; Chen et al. 2018; Djebarra et al. 2019; Ojha et al. 2019; Yin et al. 2019; Horvat et al. 2018). These six studies (Table 1) form the basis of the discussion section of our paper.

\section{Discussion}

\subsection{SUMMARY OF FINDINGS}

The following is a summary and evaluation of the critical findings which were extracted from the relevant studies.

\subsubsection{Test environment independent variables}

By using mobile body-brain sensors on participants during walking, both Chen et al. (2018) and Ojha et al. (2018) use urban scale physical environments in an attempt to understand participants' physiological responses. Horvat et al. (2018) exposed participants to virtual environments with various pictures on a wall with the intent to evoke 'happiness' or 'fear' (Horvat et al. 2018). The study conducted by Djebbara et al. (2019) tested participants' physiological reactions to affordances in transitioning from 'easily passable' and 'non-passable' spaces, in which they argue that affordances in environments impact on the perception of that environment. Banaei et al. (2017) exposed participants to various architectural styles in virtual reality by using mobile brain-body imaging and electroencephalogram while walking. Similarly, Yin et al. (2019) investigated the impacts of open and enclosed spaces by monitoring ECG, GSR, eye tracking, pulse rate and blood pressure of participants. It is evident that there are a variety of valid approaches to studying the impact of architectural space on human physiological responses.

\subsubsection{Multisensory test and experiment settings}

Four studies reported using virtual reality environments as their experimental setting (Banaei et al. 2017; Djebbara et al. 2019; Yin et al. 2019; Horvat et al. 2018), allowing participants to inhabit the space through movement using head-mounted displays. Two studies (Chen et al. 2018; Ojha et al. 2019) conduct their experiments in physical urban spaces, where natural walking is included in the tests. All of the studies which utilise virtual reality as the controlled test environment only focus on the impact of visual stimuli (Banaei et al. 2017; Djebbara et al. 2019; Yin et al. 2019; Horvat et al. 2018). Among the ones that are conducted in a physical setting, one study (Ojha et al. 2019) considers the non-visual or multisensory experience of the environment by measuring factors such as the temperature. The signals which can be interpreted 


\section{THE IMPACT OF MOVING THROUGH THE BUILT ENVIRONMENT ON EMOTIONAL AND NEUROPHYSIOLOGICAL STATE}

in the built environment are a combination of multisensory information. The tests in physical settings which are multisensory are incapable of identifying the role of each sensory input on body and brain reactions due to the extensive number of variables influencing subjects in the physical tests, and inability to control those variables in a systematic way. In regards to the current technology, acquiring a suitable statistical method for understanding the impact of simultaneous change in environmental variables is necessary.

\subsubsection{Comparing methods of data analysis}

Of the six selected studies, three used ANOVA (Banaei et al. 2017; Djebbara et al. 2019; Yin et al. 2019) as the mode of data analysis. REP-Tree (Ojha et al. 2019) and Pearson's Correlation Analysis (Horvat et al. 2018) were also implemented, with one study (Chen et al. 2018) using qualitative data analysis presented in diagrams. From this, it is evident that there is no standard for analysing data and predicting correlations; each study establishes its own parameters and explores the outcomes within the boundaries of its own scope. This means that individual case studies have different parameters and different baseline conditions, which inherently results in incompatible data. In experiments which use monitoring equipment such as EEG, ECG, fMRI and GSR, movement may cause noisy data, so is often avoided. Instead, most studies measure the physiological responses of people in static positions. It can be a limitation, as motion is a crucial part of making sense of any given haptic built environment. The majority of the six selected studies use Independent Component Analysis (ICA) to eliminate noise from raw EEG data recorded from the participants. ICA is a computational method for extracting underlying components from a multivariate signal. Hence, there is a way to mitigate the effect of movement in test scenarios; the inclusion of movement as a parameter in this field should continue to be explored in future studies. While all six studies have some form of objective data measurements such as EEG, ECG, EMG, GSR or fMRI, two studies (Ojha et al. 2019; Horvat et al. 2018) do not report any subjective self-reflection. There is undoubtedly a layer of information which can be gained from subjective self-reflection of experiences in the test environments. This inconsistency in procedure between studies makes it difficult to correlate data and make conclusions across studies.

\subsubsection{Population sample sizes and demographics}

The selected studies had a range between 4 (Chen et al. 2018) and 30 (Ojha et al. 2019; Yin et al. 2019) participants. None of the studies reports equal numbers of male and female participants, with some disproportionately favouring one of the two groups (Horvat et al. 2018; Yin et al. 2019). Two studies failed to report on the participants' gender at all (Ojha et al. 2019; Chen et al. 2018). A larger sample of data needs to be acquired in order to see whether there is even potential in claiming that physiological responses are universal. However, by analysing these few subjects, the conducted tests have been able to show statistical significant differences in body and brain reactions of the participants. Additionally, the majority of studies suggest hiring subjects that do not have extensive knowledge in the field of architecture to minimise the effect of that factor on the response. 


\subsection{LIMITATIONS OF CURRENT METHODOLOGIES}

Although a growing body of research has been published, this research field is still at the primary stages of its inception, which requires iterations of research. In future studies, more scientifically rigorous studies are needed to measure the effectiveness and accuracy of virtual reality for eliciting mental and emotional reactions akin to real-world scenarios. There is a general lack of evidence on realism within the simulations in virtual reality tests. This lack of immersion could create an element of disbelief in participants, altering the physiological reactions, and thus, the emotional responses. For example, the impact of the frame rate drop down in virtual reality setups that cause motion sickness is not discussed in the articles reviewed. Furthermore, many studies place participants in a passive role instead of an active one, and since perception is action-oriented, the way in which participants interact in the virtual environment can manipulate the body-brain interaction. By designing tests that engage people with activities in the physical or virtual environments, the outcomes may provide a more accurate dataset for understanding emotional responses. The limitations in the number of participants and the amount of data collected from them do not allow for the identification of direct interrelation between the built environment as a stimulus and the subjective and objective data of emotional reactions. A more rigorous approach is required to examine the way movement activates emotion in our experiences of built environments.

\subsection{TRAJECTORY FOR INTENDED RESEARCH}

Based on the discussion outlined above, we have identified a significant gap in the current research which requires a more robust body of evidence for conclusions to be made about the impact built environments have on emotion. We will focus on three less-investigated factors that we presume have a high potential to manipulate emotions: visual complexity, scale, and light. We argue that these factors have been utilised in the design of notable architectural buildings intuitively through history. Considering some theories of emotions which claim all emotions are capable of being mapped to a two-dimensional scale (Schachter \& Singer 1962), we will investigate how characteristics of space can evoke various emotional states such as a sense of awe in humans. We assume that by maximising emotional arousal and pleasure levels, we will be able to elicit these emotions in users of a space. As an emotion which has been evoked from architecture for millennia, understanding awe could be the next step in understanding how architecture can elicit immense physiological and emotional responses. Central to the question of which characteristics of architectural space evoke awe and what features of architectural space can produce emotional arousal? Conversely, is there a way architects can remove stress from the environment by utilising virtual environments when designing the scenarios for inhabitants to experience?

We argue that using VR would be a solution for isolating visual stimuli for scientific investigations. Architectural texts suggest the importance of movement and motion in perception and emotion (Rasmussen 1964). In recent literature, only a small number of studies have tried to measure the emotional and physiological 
responses of a build environment experience while participants are walking through those virtual or physical environments. By utilising VR, we will be able to measure and investigate the body and brain reactions of people that naturally walk in a controlled test setting. The majority of current studies suggest that multimodal biometric data and synchronisation between them contribute to the decoding the emotions of architectural experiences. Therefore, we will measure various biodata by acquiring simultaneous data from various biosensors such as EEG, GSR and Eye tracking. Our strategy for noise reduction in data includes using mathematical algorithms to eliminate noise and extract meaningful data from raw biodata. To study the interrelation between our interventions and high dimensional data gathered from biosensors, we will use advanced statistical methods such as machine learning classifications. Based on the Constructivist theory of learning, we learn through experience, which is a critical point in this matter. We have to consider that, as Piagetian theory of learning suggests (Piaget 1952), the brain may react to any external stimuli based on the culmination of all previous experiences. It means that a level of expectation in any test or experience can play an essential role in the emotional and physiological reactions. It also indicates the importance of the impact of short-term and long-term memories in future studies. We hope to investigate how expectations, or inversely, subversions of expectations in the built environment impacts physiological responses.

\section{Conclusion}

A small number of studies have emerged as a result of recent advancements in technology. These technologies pave the way for a better understanding of the interrelation between the built environment and the people who occupy it. Recent studies suggest that parameters such as open space versus enclosed space or even architectural style of an interior form can cause significant differences in neurophysiological reactions. Using portable and lightweight body-brain sensors have helped researchers to study the impact of external stimuli on people during move and stroll. It has brought a unique opportunity for researchers in the field of architecture to scientifically study emotion and come up with criteria and guidelines for the architectural design process. Moreover, modern statistical data analysis methods have helped those researchers to overcome the challenges of analyzing the raw and noisy biometric data. To conclude, since the field is still novel and anomalous, more studies need to be conducted in order to piece together the intricate way we perceive our built environment.

\section{References}

Banaei, M., Hatami, J., Yazdanfar, A. and Gramann, K.: 2017, Walking through architectural spaces: The impact of interior forms on human brain dynamics, Frontiers in Human Neuroscience, 11, 1-14

Barrie, T.: 1996, Spiritual Path, Sacred Place, Shambhala.

Bruno, G.: 2007, Atlas of Emotion: Journeys in Art, Architecture, and Film, Verso.

Cannon, W.B.: 1927, The James-Lange theory of emotions: a critical examination and an alternative theory, The American Journal of Psychology, 39, 106-124.

Chen, Z., Schulz, S., Qiu, M., Yang, W., He, X., Wang, Z. and Yang, L.: 2018, Assessing affective experience of in-situ environmental walk via wearable biosensors for 
evidence-based design, Cognitive Systems Research, 52, 970-977.

Djebbara, Z., Fich, L., Petrini, L. and Gramann, K.: 2019, Sensorimotor brain dynamics reflect architectural affordances, Proceedings of the National Academy of Sciences of the United States of America, 116(29), 14769-14778.

J.P. Eberhard (ed.): 2008, Brain Landscape: The Coexistence of Neuroscience and Architecture, Oxford University Press.

Eberhard, J.P.: 2009, Applying Neuroscience to Architecture, Neuron, 62(6), 753-756.

Gibson, J.: 1966, The Senses Considered as Perceptual Systems, Praeger.

Horvat, M., Dobrinić, M., Novosel, M. and Jerčić, P.: 2018, Assessing emotional responses induced in virtual reality using a consumer EEG headset: A preliminary report, 41st International Convention on Information and Communication Technology, Electronics and Microelectronics (MIPRO), 1006-1010.

James, W.: 1884, What is an Emotion?, Mind, 9(34), 188-205.

Kahn, L. and Brillembourg, C.: 1992, "Louis Kahn by Carlos Brillembourg [Architecture: Interview]". Available from $<$ https://bombmagazine.org/articles/louis-kahn/ $>$ (accessed 1st December 2019).

Lanzetta, J.T., Cartwright-Smith, J. and Kleck, R.E.: 1976, Effects of nonverbal dissimulation on emotional experience and autonomic arousal, Journal of Personality and Social Psychology, 33(3), 354-370.

Ojha, V.K., Griego, D., Kuliga, S., Bielik, M., Buš, P., Schaeben, C., Treyer, L., Standfest, M., Schneider, S., König, R., Donath, D. and Schmitt, G.: 2019, Machine learning approaches to understand the influence of urban environments on human's physiological response, Information Sciences, 474, 154-169.

Piaget, J.: 1952, The origins of intelligence in children, W W Norton $\backslash \&$ Co.

Picard, R.W., Vyzas, E. and Healey, J.: 2001, Toward machine emotional intelligence: analysis of affective physiological state, IEEE Transactions on Pattern Analysis and Machine Intelligence, 23(10), 1175 - 1191.

Rasmussen, S.E.: 1964, Experiencing Architecture, Second Edition, The MIT Press.

Robinson, S.: 2015, John Dewey and the dialogue between architecture and neuroscience, arq: Architectural Research Quarterly, 19(4), 361-367.

Schachter, S. and Singer, J.: 1962, Cognitive, social, and physiological determinants of emotional state, Psychological Review, 69(5), 379-399.

Schreuder, E., Erp, J.V., Toet, A. and Kallen, V.L.: 2016, Emotional Responses to Multisensory Environmental Stimuli: A Conceptual Framework and Literature Review, SAGE Open, 6(1), 1-19.

Yin, J., Arfaei, N., MacNaughton, P., Catalano, P.J., Allen, J.G. and Spengler, J.D.: 2019, Effects of biophilic interventions in office on stress reaction and cognitive function: A randomized crossover study in virtual reality, Indoor Air, 29(6), 1028-1039. 